\title{
Numerical Simulation of Sandwiched Perovskite-Based Solar Cell Using Solar Cell Capacitance Simulator (SCAPS-1D)
}

\author{
I. T. Bello ${ }^{\mathrm{a}, *}$, Y. A. Odedunmoye ${ }^{\mathrm{b}}$, O. Adedokun ${ }^{\mathrm{a}, * *}$, H. A. Shittu ${ }^{\mathrm{a}}$, A. O. Awodugba ${ }^{\mathrm{a}}$ \\ ${ }^{a}$ Department of Pure and Applied Physics, Ladoke Akintola University of Technology, Ogbomoso, Nigeria. \\ ${ }^{b}$ Department of Mathematical and Physical Sciences, Osun State University, Osogbo, Nigeria.
}

\begin{abstract}
Due to the superb characteristics of its light-harvesting, the Perovskite sensitizer $\mathrm{ABX}_{3}\left(\mathrm{~A}=\mathrm{CH}_{3} \mathrm{NH}_{3}, \mathrm{~B}=\mathrm{Pb}, \mathrm{Sn}\right.$, and $\mathrm{X}=\mathrm{Cl}, \mathrm{Br}$, I) has recently attracted great attention. Perovskite is composed of inexpensive and earth abundant materials. It is processable at low temperature preferably via the printing techniques. In addition, the charges in the bulk material after light absorption that enhances low loss in energy charge generation and collection were generated freely. In this research work, Solar cell capacitance simulator (SCAPS-1D) was used to harnessing the real device hybrid Perovskite (PSC) solar cell with material parameters obtained from literatures and experiment used in the definition panel and the arrangement of an hybrid (FTO/ZnO/CZTS/PSCS/CZTS/HTM) model in the SCAPS-1D simulator. From the simulated results obtained the Band gap diagram and other curves were constructed. The efficiency greater than twenty percent $(>20 \%)$ was achieved, which shows that having a combination of two different absorber were achievable and calling for great attention from the researchers.
\end{abstract}

Keywords: Sandwiched, Perovskite, Efficiency, Band Gap, Harnessing

Article History :

Received: 09 April 2019

Received in revised form: 22 May 2019

Accepted for publication: 27 May 2019

Published: 19 June 2019

(C)2019 Journal of the Nigerian Society of Physical Sciences. All rights reserved. Communicated by: W. A. Yahya

\section{Introduction}

The Perovskite solar cells (PSCS) originally came out as the result of unrelenting efforts on DSSC researches. The Perovskite Solar cells have become a rapidly growing area of the photovoltaic world and of huge desire to the scientific community with its improvement. Perovskite solar cells have attracted salient attention of the academic community since the first reported article in 2012 [1]. Graphene was introduced into Perovskite solar cell and an efficiency of $15.6 \%$ was obtained [2]. In $2015,20.1 \%$ efficiency was recorded when the poly-

\footnotetext{
${ }^{*}$ Corresponding author Tel. no: +2348062814778

${ }^{* *}$ Corresponding author Tel. no: $+2347031195750,+2348065976299$

Email addresses: itbello13@pgschool.lautech.edu.ng (I. T. Bello ), oadedokun@lautech.edu.ng (O. Adedokun)
}

triarylamine (PTAA) was used as a new HTM with another perovskite material, formamidinium iodide $\left(\mathrm{HC}\left(\mathrm{NH}_{2}\right)_{2} \mathrm{PbI}_{3}\right)$ [3].

There is also a vast potency for better engineering work and effective solar cells which are anticipated to reach excess power conversion efficiency (PCE) of over 20 per cent. Perovskite solar cells have increased in PCE at an unbelievably great rate in comparison with other solar cells.

Currently, the significant negative aspect of Perovskite based solar cells was not known. Although the life-times of the cells are not yet proved since there is no evidence to suggest that their life-time is any higher or less than that of pure organic devices. The use of lead in Perovskite compound is not ideal since there is potential for a lead alternative to be used in Perovskite compound instead, lead can be used in a much smaller amount than that of what is currently present in either lead or cadmium based 
batteries. Finally, the optical density of the Perovskite materials is yet to be fully discussed, although its optical density was still lower than other active materials but higher than that of silicon. As a result, the light-harvesting Perovskite devices require thicker layers which may cause some limitations in the fabrication of a solution processed devices whereby achieving high uniformity with such thick layers will be difficult.

Improvement of the precursor materials for solution based Perovskite deposition and associated coating and processing techniques will be a key development for any solution processed devices will ultimately yield lower production costs. Although at present the best Perovskite solar cells are vacuum deposited. While vacuum based processes are relatively easy to scale up, the capital equipment cost of doing so can rapidly become astronomical. To achieve a truly low cost-per-watt devices, Perovskite solar cells will require to have the much heralded trio of high efficiency, long life-times and low manufacturing costs. Perovskite based devices have so far demonstrated enormous potential for achieving this but have not yet been achieved for other thin film technologies [4]. There are many simulation software models used to simulate solar cells devices numerically, such as Large-scale Atomic/Molecular Massively Parallel Simulator (LAMMPS), Silvaco ATLAS, Solar Cell Capacitance Simulator (SCAPS) etc. In this research work, SCAPS software will be used to simulate a Perovskite based solar cell. SCAPS (Solar Cell Capacitance Simulator) is a one-dimensional simulation program with seven semiconductor input layers developed by a group of Photovoltaic researcher at the Department of Electronics and Information System, University of Gent, Belgium [5].

\section{Device Structure}

The cell model used in the simulation is $\mathrm{n}-\mathrm{FTO} / \mathrm{n}-\mathrm{ZnO} / \mathrm{p}-$ CZTS/p-PSCS/p-CZTS/HTM. This cell structure consists of Fluorine doped Tin Oxide $\left(\mathrm{Sn}_{2} \mathrm{O}: \mathrm{F}\right)$, as the window layer, namely, a conductive n-type $\mathrm{ZnO}$, Perovskite $\left(\mathrm{CH}_{3} \mathrm{NH}_{3} \mathrm{~Pb}_{3}\right.$-xClx) and a $\mathrm{Cu}_{2} \mathrm{ZnSnS}_{4}$ (CZTS) which are p-type semiconductors. Figure 1. shows the solar cells layers structure. The cell illuminated through the Transparent conductive oxide (TCO), which serves as a window layer, passes across the electron transport layer (ntype $\mathrm{ZnO}$ ) which serves as a buffer layer and enters the absorber layer to the hole transport material.

\section{Methodology and Simulations}

Numerical simulation technique of solar cells devices has over the years proved to be a viable tool for studying and understanding the properties of solar cell devices such as the optical, electrical and mechanical properties of complex solar cell devices [5]. It also helps to reduce processing cost and time spent on solar cell device fabrication by providing useful information on how to vary the production parameters to improve the device performance [7][8]. SCAPS-1D simulator based its simulations on the solutions of the three basics semiconductor equations; Poissons equation, continuity equation of electron and continuity equation of hole. SCAPS-1D software solves these three

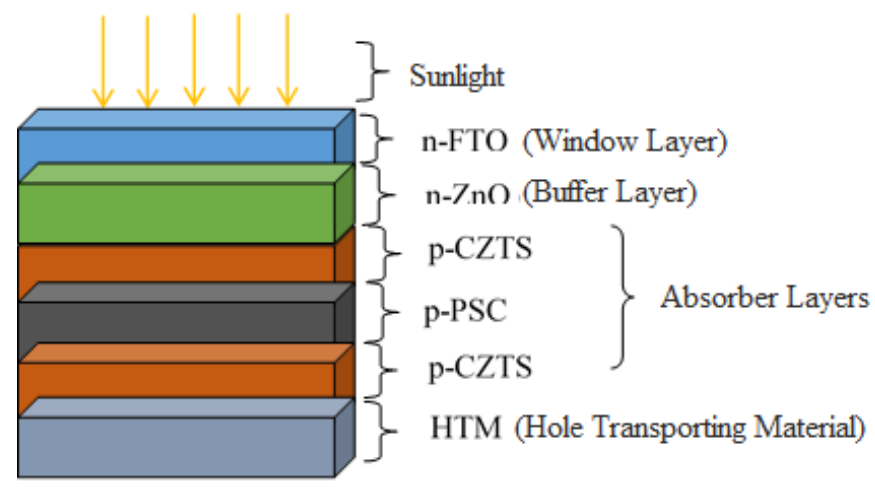

Figure 1: Model of Sandwiched Simulation structure [6].

coupled partial differential equations numerically for the electrostatic potentials electron and hole concentration as a function of positions $\mathrm{x}$.

Poisson's equation is given as

$$
\frac{\partial}{\partial x}\left(\epsilon \frac{\partial \Psi}{\partial x}\right)=\frac{-q}{\epsilon_{0}}\left[p-n+N_{D}^{+}-N_{A}^{-}+\frac{\rho d e f(n, p)}{q}\right]
$$

where $\Psi$ is electrostatic potential, $\epsilon$ is dielectric constant and $\mathrm{q}$ is an electronic charge. The first two terms in the right are free charge carriers per volume, third and fourth are ionized donor and acceptor-like dopants i.e, localized states and $\rho$ defis defect charge density. Thus, the conservation of free electrons and free holes in the device is expressed as continuity equations

$$
\begin{aligned}
& \frac{\partial n}{\partial t}=-\frac{\partial J_{n}}{\partial x}+G-U_{n}(n, p) \\
& \frac{\partial p}{\partial t}=-\frac{\partial J_{p}}{\partial x}+G-U_{p}(n, p)
\end{aligned}
$$

where P, $\mathrm{n}$ - free carrier concentrations, $N_{D, A}$ - charged dopants, $\rho \operatorname{de} f(n, p)-$ defect distributions, $j_{n}, j_{p}^{-}$the electron and hole current densities, $U_{n, p^{-}}$the net recombination rates, G- the generation rate.

SCAPS-1D was used in this work to harnessing the real device hybrid Perovskite (PSC) solar cell with material parameters defined in Table 1.0 which were used in the definition panel of the SCAPS-1D simulator. The absorption coefficients of the materials used were determined by the simulator based on the input parameters (Table 1.0) and the arrangement of the model as allowed by the SCAPS-1D simulator. From Table 1.0, shown above, absorber layers were varied while the other parameters are kept constant. Various efficiencies were generated based on the thickness variation of the absorber. All simulations in this work were performed under ambient temperature (300K). The electrical parameters $\left(V_{O C}, J_{S C}, F F\right)$ and efficiency generated by SCAPS-1D would then be used to determine the optimum thickness of the absorber layer. From, this, the J-V, C-V, C-f and Q.E of the best solar cells from the simulation will be determined and the effect of sandwich in the solar cell. 
Table 1: Materials parameter used in simulation [7][8][9].

\begin{tabular}{lcccr}
\hline Parameters & FTO & ZnO & PSC & CZTS \\
\hline Thickness $(\mu \mathrm{m})$ & 0.5 & 0.05 & varied & varied \\
Band Gap $(\mathrm{eV})$ & 3.5 & 3.35 & 1.55 & 1.55 \\
Electron affinity $(\mathrm{eV})$ & 4.0 & 4.21 & 3.9 & 4.5 \\
Dielectric constant & 9 & 9 & 6.5 & 10 \\
Conduction Band-DOS $N_{c}\left(\mathrm{~cm}^{-3}\right)$ & $2.2 .10^{18}$ & $2.2 .10^{18}$ & $2.2 .10^{18}$ & $2.2 .10^{18}$ \\
Valence Band-DOS $N_{v}\left(\mathrm{~cm}^{-3}\right)$ & $1.8 .10^{19}$ & $1.8 .10^{19}$ & $1.8 .10^{19}$ & $1.8 .10^{19}$ \\
Electron Thermal Velocity $(\mathrm{cm} / \mathrm{s})$ & $1.0 .10^{7}$ & $1.0 .10^{7}$ & $3.0 .10^{7}$ & $1.0 .10^{7}$ \\
Hole Thermal Velocity $(\mathrm{cm} / \mathrm{s})$ & $1.0 .10^{7}$ & $1.0 .10^{7}$ & $3.0 .10^{7}$ & $1.0 .10^{7}$ \\
Electron mobility $\mathrm{cm}^{-3} \cdot \mathrm{V}^{-1} \cdot \mathrm{s}^{-1}$ & 2.0 & 25 & 1.6 & 100 \\
Hole mobility $\mathrm{cm}^{-3} \cdot \mathrm{V}^{-1} \cdot \mathrm{s}^{-1}$ & 1.0 & 100 & 0.2 & 20 \\
Donor density $N_{D}\left(\mathrm{~cm}^{-3}\right)$ & $2.0 .10^{19}$ & $1.0 .10^{18}$ & 0 & 0 \\
Acceptor density $N_{A}\left(\mathrm{~cm}^{-3}\right)$ & 0 & 0 & $6.10^{18}$ & $8.22 .10^{18}$ \\
\hline
\end{tabular}

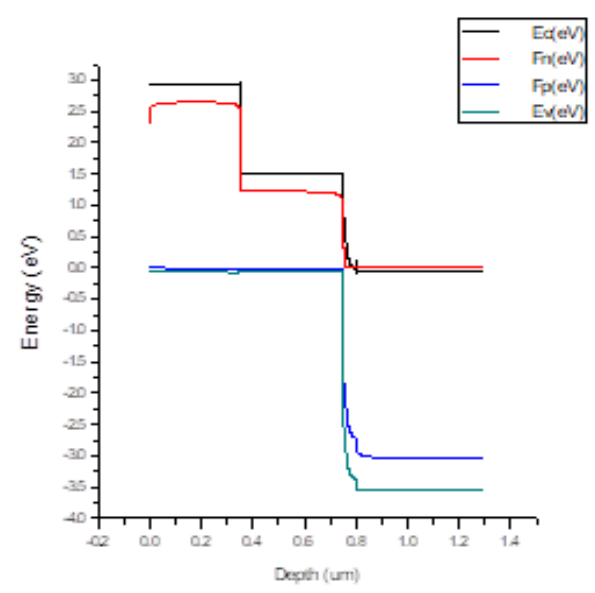

(a)

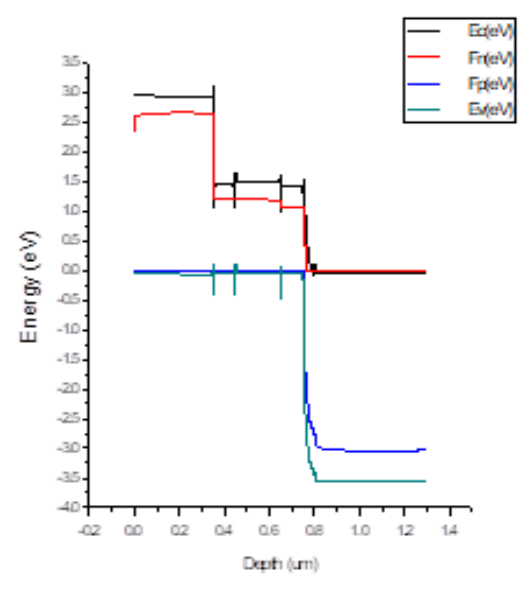

(b)

Figure 2: (a) The Band diagram of Perovskite. (b) The Band diagram of sandwiched Perovskite

\section{Results and Discussion}

\subsection{The Bandgap diagram}

Figure 2, show the band diagram of the sandwiched Perovskite device. The band gap line up model of the simulated device of an hybrid FTO/ZnO/CZTS/PSCS/CZTS/HTM was constructed from the data obtained from the SCAPS under the ambient temperature (300K).

The band diagram of Perovskite depends on the compositional variation of the component entails in the processing and synthesis of the absorber materials such as organic, metal and anion composition of the material. The band gap of the absorbing material is a crucial parameter for Photovoltaic actions, as the absorber layer is the key material in any solar cell devices [10]. Thus the band alignment is the Type II Broken band gap with a band gap of approximately $1.55 \mathrm{eV}$ which is concurrent with the theoretical condition as reported by [11]. However, it was shown from Figures $2 \mathrm{a}$ and $2 \mathrm{~b}$ above, that the band alignment of Perovskite solar cells shows single junction in the band gap while that of sandwiched Perovskite band gap shows three junctions which confirmed the presence of a sandwiching materials embedded within the absorber layer.

\subsection{J-V Curve characteristic of simulated device}

$\mathrm{J}-\mathrm{V}$ curves are the parameters used to determine the electrical output power of any solar cells. The J-V curve characteristic was obtained with the simulation of data in the Table 1.0 was shown in Figure 3 with Open Circuit Voltage $(\mathrm{Voc})=$ $0.80 \mathrm{~V}$, Short Circuit Current $(\mathrm{Jsc})=25.12 \mathrm{~mA} / \mathrm{cm}^{2}$, Fill Factor $(\mathrm{FF})=49.99 \%$ and Percentage conversion Efficiency $(\mathrm{PCE})=$ $20.09 \%$ as the cell output parameters under the standard simulated sunlight of AM1.5G and working conditions of ambient temperature and frequency of $106 \mathrm{~Hz}$.

\subsection{Effect of Variation in the sandwiched Absorber Layer thick- ness}

Solar cell absorber layer plays an important roles in the fabrication and harnessing optimum values of the solar cell efficiencies. Solar cell device performance depends solely on the electrical characteristic and variation of the absorber thickness. Thereby this research simulation work tries to fix out the effect 


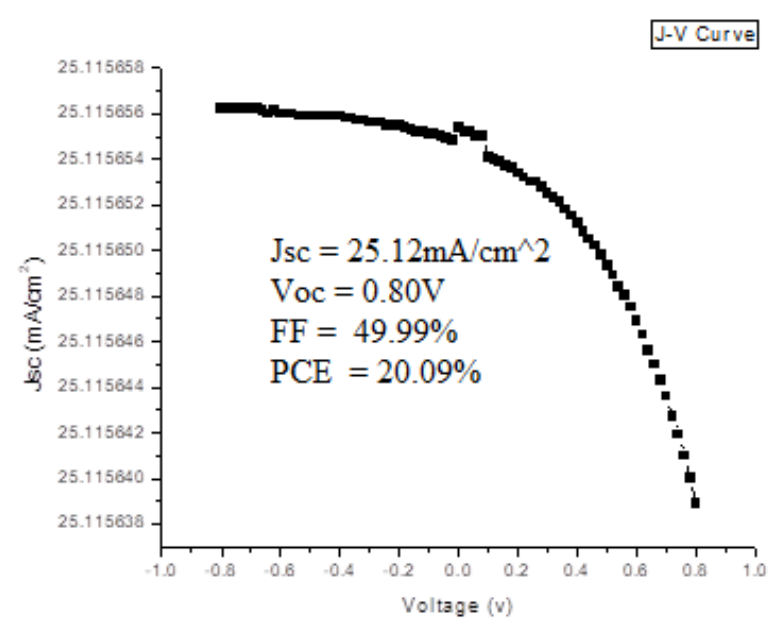

Figure 3: Sandwich I-V Curve Characteristic

of the combination of CZTS absorber layer and organometallic (Perovskite) layer, embedded in one solar cell. It was observed that the short circuit current and percentage conversion efficiency of the sandwiched absorber solar cell were $25.12 \mathrm{~mA} / \mathrm{cm} 2$ and $20.09 \%$ which are higher than the simulated Perovskite device output in this work. From Figure 4, it was observed that

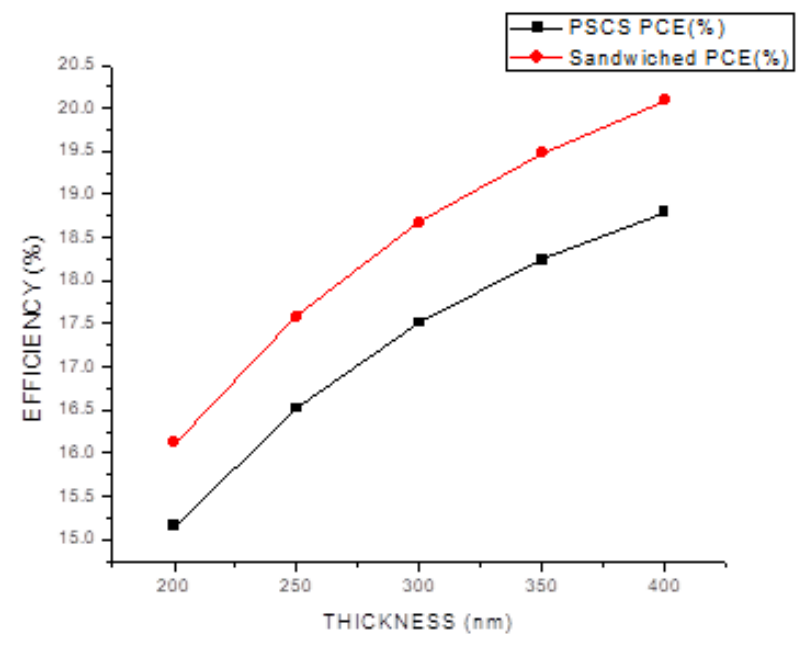

Figure 4: PSCS and Sandwiched PCE against Thickness

at $200 \mathrm{~nm}$ the efficiency of Perovskite is around $15.2 \%$ while that of sandwiched Perovskite was greater than $16.15 \%$ efficiency. Also, at $250 \mathrm{~nm}$ the efficiencies of $16.52 \%$ and $17.58 \%$ were of observed for Perovskite and sandwiched Perovskite respectively. At $300 \mathrm{~nm}$, an efficiency of $17.51 \%$ was observed for Perovskite solar cell while $18.57 \%$ was observed for sandwiched Perovskite. However, $18.24 \%$ and $19.48 \%$ efficiencies were observed at $350 \mathrm{~nm}$ for both Perovskite and sandwiched Perovskite solar cells respectively. Lastly, at $400 \mathrm{~nm}$ the efficiency of Perovskite was observed to be $18.79 \%$ and that of sandwiched Perovskite was around $20.09 \%$. Thus, the appre-

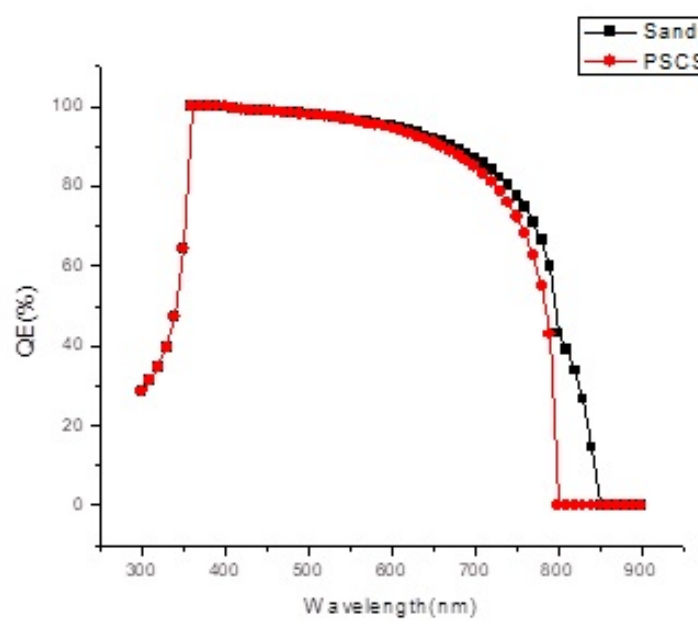

Figure 5: PSCS and Sandwiched QE against Wavelength

ciable increment in the efficiencies values of sandwiched Perovskite has shown the positive effects of sandwiching absorber layer the solar cells.

\subsection{Quantum efficiency of the solar cell}

The quantum efficiency is the ratio of the number of carriers collected by the solar cell to the number of photons of a given energy incident on the solar cell. However, Quantum efficiency is the fraction of the excited carriers that combine radiatively to the total recombination. Figure 5 is the Q.E plot against the wavelength which showed that more than $90 \%$ of the wavelength between $300 \mathrm{~nm}$ and $890 \mathrm{~nm}$ radiatively recombine and less than $10 \%$ of such wavelength recombined through other processes (Auger and SRH). The results implied that, at the $400 \mathrm{~nm}$ thickness, sandwiched layer absorbs almost all the incident photons to create the electron-hole pairs and the photogenerated carriers are almost separated and transported to the Hole transport materials and electron transport material by the built-in field with minimum recombination. Therefore it can be considered that higher thickness is the optimal length of photovoltaic action. The quantum efficiency may be given either as a function of wavelength or as energy.

Figure 5, showed that sandwiched layer can absorb incident photons up to $800 \mathrm{~nm}$, which implied that sandwiched absorber layer can perform better than Perovskite layer which can only absorb photons around $750 \mathrm{~nm}$ because of the higher the wavelength the lower photon energy.

\section{Conclusion}

In conclusion, Perovskite and Sandwiched Perovskite-Based solar cell has been successfully simulated using One-Dimensional Solar Cell Capacitance Simulator (SCAPS-1D). The output results of the simulation were recorded and plotted across the thickness variation of the absorber layers which varies from 
$200 \mathrm{~nm}$ to $400 \mathrm{~nm}$. It was found out that the higher the absorber thickness the higher the efficiencies and other electrical parameters output in the solar cell. The efficiencies of $18.79 \%$ and $20.09 \%$ were recorded for the Perovskite and sandwiched Perovskite-Based solar cells respectively.

\section{Acknowledgments}

The authors would like to appreciate Prof. Marc. Burgelman and his co-researchers at the University of Gents, Belgium for making SCAPS-1D available for use. One of the authors also thanks the TETFUND for grant used in this work and others. The authors also thank the referees for the positive enlightening comments and suggestions, which have greatly helped them in making improvements to this paper.

\section{References}

[1] M. M. Lee, J. Teuscher, T. Miyasaka, T. N. Murakami \& H. J. Snaith "Efficient hybrid solar cells based on meso-superstructured organometal halide perovskites", Science 338 (2012) 643.
[2] J. T. W. Wang, J. M. Ball, E. M., Barea, A. Abate, J. A. AlexanderWebber, J. Huang, M. Saliba, I. Mora-Sero, J. Bisquert \& H. J. Snaith "Low-temperature processed electron collection layers of graphene/TiO2 nanocomposites in thin film PSCs", Nano Letter 14 (2014) 724

[3] W. S. Yang, J. H. Noh, N. J. Jeon, Y. C. Kim, S. Ryu, J. Seo \& S. I. Seok, "High-performance photovoltaic Perovskite layers fabricated through intramolecular exchange", Science 348 (2015) 1234.

[4] http://www.ossila.com/pages/Perovskites-and-Perovskite-solar-cells-anintroduction (accessed December, 2017).

[5] A. Niemegeers, M. Burgelman, K. Decock, J. Verschraegen \& S. Degrave, "SCAPS Manual", University of Gent, 2014.

[6] I. T. Bello, M. K. Awodele, O. Adedokun, O. Akinrinola \& A. O. Awodugba, "Modelling and simulation of CZTS-Perovskite sandwiched tandem solar cell", Turkish Journal of Physics 42 (2018) 321.

[7] M. Takashi \& M. Masashi, "Device modelling of Perovskite solar cells based on structural similarity with thin film inorganic semiconductor solar cells", Journal of Applied Physics 116 (2014) 054505

[8] M. Takashi \& M. Masashi, "Theoretical analysis on effect of band offsets in Perovskite solar cells", Solar Energy Materials \& Solar Cells 133 (2015) 8 .

[9] M. Gloeckler, A. L. Fahrenbruch \& J. R. Sites, "Numerical modeling of CIGS and CdTe solar cells: setting the baseline", In Proc. 3rd World Conf. Photovoltaic Energy Conversion, 2003 pp 491.

[10] S.J. Fonash "Solar Cell Device Physics", 2nd edition Elsevier, USA; 2010.

[11] W. Shockley \& H. J. Queisser "Detailed Balance Limit of Efficiency of pn Junction Solar Cells”, Journal of Applied Physics 32 (1961) 510. 